\title{
Generalized Cantor Set and its Fractal Dimension
}

\author{
M. J. Islam ${ }^{a}$ and M. S. Islam ${ }^{b^{*}}$ \\ ${ }^{a}$ Department of Mathematics, Bangladesh Institute of Science and Technology, Bangladesh and \\ ${ }^{b}$ Department of Mathematics, University of Dhaka, Dhaka-1000, Bangladesh
}

\begin{abstract}
In this article, we discuss the Cantor set and its fractal dimension. We show the Cantor middle $\frac{1}{5}, \frac{1}{7}, \frac{1}{9}, \Lambda$ set, in general, Cantor middle $\frac{1}{2 m-1}$ which is generalized Cantor set, where $2 \leq m<\infty$. We also generalized the box-counting dimension of Cantor set that is $\frac{\ln m}{\ln (2 m-1)}$, where $2 \leq m<\infty$.
\end{abstract}

Key Words: Cantor set, fractal, fractal dimension, box-counting dimension.

\section{Introduction}

Cantor sets were discovered by the German Mathematician George Cantor in the late 19th to early 20th centuries (18451918). He introduced fractal which has come to be known as the Cantor set, or Cantor dust. The set has some interesting properties which have led to further research and discovery in fractals and chaos theory (Richard, 1996).

Fractal is defined by B. Mandelbrot is a shape made of parts similar to the whole in some way in the 1960's. Fractals can be classified in numerous manners, of which one stands out rather distinctly, exact (regular) fractals versus statistical (random) fractals. An exact fractal is an object which appears self-similar under varying degrees of magnification in effect, possessing symmetry across scale with each small part replicating the structure of the whole. Statistical fractals have been observed in many physical systems, ranging from material structures (polymers, aggregation, interfaces, etc.) to biology, medicine, electric circuits, computer interconnects, galactic clusters, and many other surprising areas, including stock market price fluctuations (Addison, 1997).

Fractal is a geometric object that possesses the two properties: self-similar and non-integer dimensions. So a fractal is an object or quantity which displays self-similarity.

George Cantor found the Cantor middle $\frac{1}{3}$ set. Kathleen T. of the Cantor middle $\frac{1}{3}$ set. We show Cantor middle $\frac{1}{5}$, $\frac{1}{7}, \frac{1}{9}, \frac{1}{11}, \Lambda$ set, in general, Cantor middle $\frac{1}{2 m-1}$ set, where $2 \leq m<\infty$. Also we find the box-counting dimension of them and generalize the box-counting dimension of the Cantor set. The generalized box-counting dimension of the Cantor set is $\frac{\ln m}{\ln (2 m-1)}$, where $2 \leq m<\infty$.

\section{Generalization of Cantor set}

\section{Definition}

A nom empty set $\Gamma \subset \mathbf{R}$ is called a Cantor set if

(a) $\Gamma$ is closed and bounded.

(b) $\Gamma$ contains no intervals.

(c) Every point in $\Gamma$ is an accumulation point of $\Gamma$.

The Cantor middle $\frac{1}{3}$ set which is created by the following algorithm:

We start with the closed interval $[0,1]$. Call this set $\Gamma_{0}$.

Alligood et. al. (1997) found the box-counting dimension

*Corresponding author. E-mail: jahurul93@gmail.com, mshahidul11@yahoo.com 
G

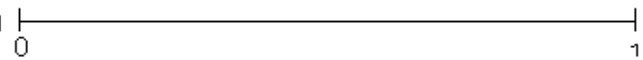

Remove the middle open third This leaves a new set, called $\Gamma_{1}$, which is $\left[0, \frac{1}{3}\right] \cup\left[\frac{2}{3}, 1\right]$.
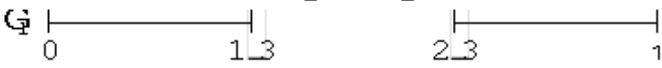

Each iteration through the algorithm removes the open middle third from each segment of the previous iteration. Thus the next two sets would be

$$
\begin{aligned}
& \Gamma_{2}=\left[0, \frac{1}{9}\right] \cup\left[\frac{2}{9}, \frac{1}{3}\right] \cup\left[\frac{2}{3}, \frac{7}{9}\right] \cup\left[\frac{8}{9}, 1\right] . \\
& \dot{4} \vdash_{19} \underbrace{1}_{9} 3
\end{aligned}
$$

and $\Gamma_{3}=\left[0, \frac{1}{27}\right] \cup\left[\frac{2}{27}, \frac{1}{9}\right] \cup\left[\frac{2}{9}, \frac{7}{27}\right] \cup\left[\frac{8}{27}, \frac{1}{3}\right]$

$$
\cup\left[\frac{2}{3}, \frac{19}{27}\right] \cup\left[\frac{20}{27}, \frac{7}{9}\right] \cup\left[\frac{8}{9}, \frac{25}{27}\right] \cup\left[\frac{26}{27}, 1\right] \text {. }
$$

$$
\text { ษ户 }
$$

In general, after $\boldsymbol{n}$ times iterations, we obtain $\Gamma_{n}$ which as follows

$\Gamma_{n}=\left[0, \frac{1}{3^{n}}\right] \cup\left[\frac{2}{3^{n}}, \frac{3}{3^{n}}\right] \cup \Lambda$

$\cup\left[\frac{3^{n}-3}{3^{n}}, \frac{3^{n}-2}{3^{n}}\right] \cup\left[\frac{3^{n}-1}{3^{n}}, 1\right]$, where $n \geq 0$

The Cantor set is defined to be the set of the points that remain as the number of iterations tends to infinity. The Cantor middle $\frac{1}{3}$ set is the set $\Gamma=\coprod_{n=0}^{\infty} \Gamma_{n}$.

We can show that the total length of the segments removed is 1 . Note that in the first iteration we removed $1 / 3$, in the second iteration we removed $2 / 9$, in the third iteration we removed $4 / 27$, and in the fourth iteration we removed $8 / 81$, and so forth. This is a geometric series with first term $a=\frac{1}{3}$ and common ratio $r=\frac{2}{3}$. This converges, and the sum is $S_{\infty}=\frac{1 / 3}{1-2 / 3}=1$. Any point that is an endpoint of an interval at any iteration survives and is a member of the Cantor set.

The Cantor middle $\frac{1}{5}$ set which is created by the following algorithm:

We start with the closed interval $[0,1]$. Call this set $\Gamma_{0}$.

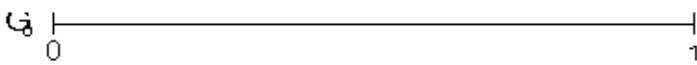

Remove the middle open interval $(1 / 5,2 / 5)$ and $(3 / 5,4 / 5)$. This leaves a new set, called $\Gamma_{1}$, which is $\left[0, \frac{1}{5}\right] \cup\left[\frac{2}{5}, \frac{3}{5}\right] \cup\left[\frac{4}{5}, 1\right]$
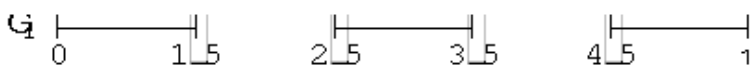

Each iter ation through the algorithm removes the open 2nd and 4th interval from each segment of the previous iteration. Thus the next set would be

$$
\begin{aligned}
& \Gamma_{2}=\left[0, \frac{1}{25}\right] \cup\left[\frac{2}{25}, \frac{3}{25}\right] \cup\left[\frac{4}{25}, \frac{1}{5}\right] \cup\left[\frac{2}{5}, \frac{11}{25}\right] \cup\left[\frac{12}{25},\right. \\
& \left.\frac{13}{25}\right] \cup\left[\frac{14}{25}, \frac{3}{5}\right] \cup\left[\frac{4}{5}, \frac{21}{25}\right] \cup\left[\frac{22}{25}, \frac{23}{25}\right] \cup\left[\frac{24}{25}, 1\right] . \\
& \dot{1} \mapsto \mapsto \mapsto_{1}
\end{aligned}
$$

In general, after $\boldsymbol{n}$ times iterations, we obtain $\Gamma_{n}$ which as follows

$\Gamma_{n}=\left[0, \frac{1}{5^{n}}\right] \cup\left[\frac{2}{5^{n}}, \frac{3}{5^{n}}\right] \cup \Lambda$

$\cup\left[\frac{5^{n}-3}{5^{n}}, \frac{5^{n}-2}{5^{n}}\right] \cup\left[\frac{5^{n}-1}{5^{n}}, 1\right]$, where $n \geq 0$.

The Cantor middle $\frac{1}{5}$ set is the set $\Gamma=\coprod_{n=0}^{\infty} \Gamma_{n}$.

We can show that the total length of the segments removed is 1 . Note that in the first iteration we removed $2 / 5$, in the second iteration we removed $6 / 25$, in the third iteration we removed $18 / 125$, and so forth. This is a geometric series with with first term $a=\frac{2}{5}$ and common ratio $r=\frac{3}{5}$. This converges, and the sum is $S_{\infty}=\frac{2 / 5}{1-3 / 5}=1$.

The Cantor middle $\frac{1}{7}$ set which set is created by the following algorithm:

We start with the closed interval $[0,1]$. Call this set $\Gamma_{0}$.

G

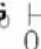


Remove the middle open interval $(1 / 7,2 / 7)$, $(3 / 7,4 / 7)$, and $(5 / 7,6 / 7)$. This leaves a new set, called $\Gamma_{1}$, which is $\left[0, \frac{1}{7}\right] \cup\left[\frac{2}{7}, \frac{3}{7}\right] \cup\left[\frac{4}{7}, \frac{5}{7}\right] \cup\left[\frac{6}{7}, 1\right]$.

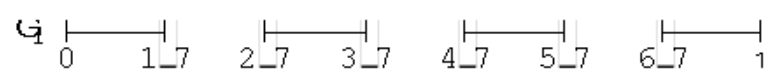

Each iteration through the algorithm removes the open 2nd, 4th, and 6th interval from each segment of the previous iteration. In general, after $\boldsymbol{n}$ times iterations, we obtain $\Gamma_{n}$ which as follows

$\Gamma_{n}=\left[0, \frac{1}{7^{n}}\right] \cup\left[\frac{2}{7^{n}}, \frac{3}{7^{n}}\right] \cup \Lambda$

$\cup\left[\frac{7^{n}-3}{7^{n}}, \frac{7^{n}-2}{7^{n}}\right] \cup\left[\frac{7^{n}-1}{7^{n}}, 1\right]$, where $n \geq 0$.

The Cantor middle $\frac{1}{7}$ set is the set $\Gamma=\mathrm{I}_{n=0}^{\infty} \Gamma_{n}$.

We can show that the total length of the segments removed is 1 . Note that in the first iteration we removed $3 / 7$, in the second iteration we removed $12 / 49$, in the third iteration we removed $48 / 343$ and so forth. This is a geometric series with first term $a=\frac{3}{7}$ and common ratio $r=\frac{4}{7}$. This converges, and the sum is $S_{\infty}=\frac{3 / 7}{1-4 / 7}=1$.

From the above construction, we can construct the Cantor middle $\frac{1}{9}, \frac{1}{11}, \frac{1}{13}, \Lambda$ set, in general, the Cantor middle $\frac{1}{2 m-1}$ set.

If $0<\frac{1}{2 m-1}<1$, where $2 \leq m<\infty$, then we can construct similar sets called Cantor middle $\frac{1}{2 m-1}$ sets by removing an interval from the center of each remaining interval whose length is $\frac{1}{2 m-1}$ times the length of the remaining interval.

The Cantor middle $\frac{1}{2 m-1}$ set which is created by the following algorithm:

We start with the closed interval $[0,1]$. Call this set $\Gamma_{0}$.

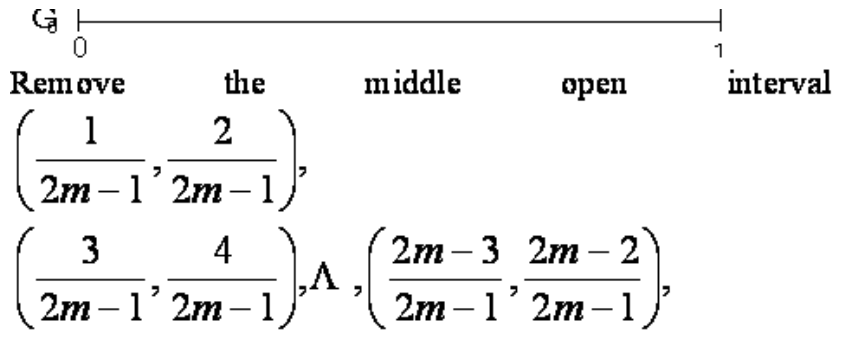

where $2 \leq \boldsymbol{m}<\infty$. In general, after $\boldsymbol{n}$ times iterations, we obtain $\Gamma_{n}$ which is as follows

$$
\begin{aligned}
& {\left[0, \frac{1}{(2 m-1)^{n}}\right] \cup\left[\frac{2}{(2 m-1)^{n}}, \frac{3}{(2 m-1)^{n}}\right] \cup \Lambda} \\
& \cup\left[\frac{(2 m-1)^{n}-3}{(2 m-1)^{n}}, \frac{(2 m-1)^{n}-2}{(2 m-1)^{n}}\right] \cup\left[\frac{(2 m-1)^{n}-1}{(2 m-1)^{n}}, 1\right],
\end{aligned}
$$

where $n \geq 0$, and $m \geq 2$. The Cantor middle $\frac{1}{2 m-1}$ set is the set $\Gamma=\mathrm{I}_{n=0}^{\infty} \Gamma_{n}$.

2.2 Lemma: If $\Gamma_{n}$ is defined in Cantor middle $\frac{1}{2 m-1}$ set, where $2 \leq m<\infty$, then there are $m^{n}$ closed intervals in $\Gamma_{n}$ and the length of each closed interval is $\left(\frac{1-\frac{1}{2 m-1}}{2 m-2}\right)^{n}$, where $2 \leq m<\infty$. Also the combined length of the intervals in $\Gamma_{n}$ is $\left(\frac{m}{2 m-1}\right)^{n}$, where $2 \leq m<\infty$, which is approaches to zero as $n$ approaches to infinity.

Proof: We start with the interval $[0,1]$ whose length is 1 . We proceed by mathematical induction. In the first step, we remove a gape of length $\frac{1}{2 m-1}$ and obtain $m$ closed intervals whose combined length is $\frac{m}{2 m-1}$. So each interval has a length of $\left(\frac{1-\frac{1}{2 m-1}}{2 m-2}\right)$, where $2 \leq \boldsymbol{m}<\infty$. In general, suppose that there are $\boldsymbol{m}^{k}$ intervals remain in $\Gamma_{k}$, each with a length of 
$\left(\frac{1-\frac{1}{2 m-1}}{2 m-2}\right)^{k}$, where $2 \leq m<\infty$, for a combined length of $\left(\frac{m}{2 m-1}\right)^{k}$, where $2 \leq m<\infty$. We will show that there are $m^{k+1}$ intervals remain in $\Gamma_{k+1}$, each with a length $\left(\frac{1-\frac{1}{2 m-1}}{2 m-2}\right)^{k+1}$, where $2 \leq m<\infty$, for a combined length $\left(\frac{m}{2 m-1}\right)^{k+1}$, where $2 \leq \boldsymbol{m}<\infty$. Note that each time we remove the middle $\frac{1}{2 m-1}$, where $2 \leq m<\infty$, portion of a closed interval s, we split the interval into $\boldsymbol{m}$ closed intervals. So in passing from $\Gamma_{k}$ to $\Gamma_{k+1}$, we multiple the number of intervals by $\boldsymbol{m}$, and there are $\boldsymbol{m}\left(\boldsymbol{m}^{k}\right)=\boldsymbol{m}^{k+1}$, where $\mathbf{2} \leq \boldsymbol{m}<\infty$, intervals in $\Gamma_{k+1}$. By assumption, each interval in $\Gamma_{k}$ has a length of $\left(\frac{1-\frac{1}{2 m-1}}{2 m-2}\right)^{k}$, where $2 \leq m<\infty$. Since we remove the middle $\frac{1}{2 m-1}$, where $2 \leq m<\infty$, portion of each interval in $\Gamma_{k}$ to create $\Gamma_{k+1}$, the amount of each interval from $\Gamma_{k}$ left in $\Gamma_{k+1}$ is

$$
\begin{aligned}
& \left(\frac{1-\frac{1}{2 m-1}}{2 m-2}\right)^{k}-\frac{1}{2 m-1}\left(\frac{1-\frac{1}{2 m-1}}{2 m-2}\right)^{k} \\
& =\frac{\left(1-\frac{1}{2 m-1}\right)^{k+1}}{(2 m-2)^{k}}, \text { where } 2 \leq m<\infty \text {. As this length }
\end{aligned}
$$
is left in $2 m-2$ intervals, the length of each remaining interval $\frac{1}{2 m-2} \times \frac{\left(1-\frac{1}{2 m-1}\right)^{k+1}}{(2 m-2)^{k}}=\left(\frac{1-\frac{1}{2 m-1}}{2 m-2}\right)^{k+1}$, where $2 \leq \boldsymbol{m}<\infty$. Finally, there are $\boldsymbol{m}^{k+1}$ intervals in $\Gamma_{k+1}$, so the combined length of the intervals in $\Gamma_{k+1}$ is

$$
\begin{aligned}
& \boldsymbol{m}^{k+1} \times\left(\frac{1-\frac{1}{2 m-1}}{2 m-2}\right)^{k+1}=\left(\frac{m}{2 m-1}\right)^{k+1}, \quad \text { where } \\
& 2 \leq \boldsymbol{m}<\infty \text {. Since } \quad 0<\frac{1}{2 m-1}<1,\left(\frac{m}{2 m-1}\right)^{n}
\end{aligned}
$$

converges to 0 as $n$ grows without bound and it follows that the combined length of the intervals in $\Gamma_{n}$ approaches 0 as $n$ goes to infinity.

\section{Proposition}

The Cantor middle $\frac{1}{2 m-1}$ set is a Cantor set, where $2 \leq m<\infty$.

Proof

Let $\Gamma$ be a Cantor middle $\frac{1}{2 m-1}$ set, where $2 \leq \boldsymbol{m}<\infty$. Since 0 is in every $\Gamma_{n}, \Gamma$ is not empty. To complete the proof, we must show that (i) $\Gamma$ is closed and bounded, (ii) $\Gamma$ contains no intervals, and (iii) every point of $\Gamma$ is an accumulation point of $\Gamma$.

(i) Since $\Gamma$ is the intersection of closed intervals, it is closed As $\Gamma$ is contained in $[0,1]$, it is also bounded

(ii) If $\Gamma$ contains an open interval $(x, y)$ with length $|\boldsymbol{y}-\boldsymbol{x}|$, then at each stage in the construction of $\Gamma,(x, y)$ must be contained in one of the remaining closed intervals. However, Lemma 2.2 implies that after $n$ steps the length of one of these intervals is $\left(\frac{1-\frac{1}{2 m-1}}{2 m-2}\right)^{n}$, where $2 \leq m<\infty$, and we can find an 
$\boldsymbol{n}_{0}$ such that $\left(\frac{1-\frac{1}{2 m-1}}{2 m-2}\right)^{n_{0}}<|y-x|$, where $2 \leq m<\infty$, that is, the length of each of the closed intervals in $\Gamma_{n_{0}}$ is less than the length of $(x, y)$. Hence, the entire interval $(x, y)$ cannot be contained in $\Gamma_{r_{0}}$ and $\Gamma$ contains no intervals.

(iii) Suppose that $\boldsymbol{x}$ is a point in $\Gamma$ and let $N_{\varepsilon}(x)=(x-\varepsilon, x+\varepsilon)$ be a neighborhood of $x$. We must show that there exists a point in $\Gamma$ that is contained in $N_{\varepsilon}(x)$ and is not equal to $x$. Notice that if $x_{1}$ is an endpoint of one of the intervals that is removed, then $x_{1}$ is in $\Gamma$. Now at each stage in the construction of the Cantor set, $\boldsymbol{x}$ must be in one of the remaining closed intervals. That is, for each $\boldsymbol{n}$ there is an intervals in $\Gamma_{n}$ that contains $x$. Choose $n$ large enough so that $\left(\frac{1-\frac{1}{2 m-1}}{2 m-2}\right)^{n}<\varepsilon$, where $2 \leq m<\infty$. Then $x$ is in one of the closed intervals that comprise $\Gamma_{n}$. Call this interval $I_{n}$. By Lemma 2.2, the length of $I_{n}$ is $\left(\frac{1-\frac{1}{2 m-1}}{2 m-2}\right)^{n}, \quad$ where $\quad 2 \leq m<\infty . \quad$ Since
$\left(\frac{1-\frac{1}{2 m-1}}{2 m-2}\right)^{n}<\varepsilon$, where $2 \leq m<\infty$, it must be the endpoints of $I_{n}$ are in $N_{\varepsilon}(x)$. As there are two endpoints and $x$ can be equal to at one of them, other endpoint is an accumulation point of $\Gamma$. The proof is complete.

\section{Fractal Dimension}

Consider a grid of step-size $1 / \boldsymbol{n}$ on the unit interval $[0,1]$, that is, there are grid points at
$0,1 / n, 2 / n, \ldots .(n-1) / n, 1$. That is there are $n$ boxes of grid of size $1 / n$. The situation changes slightly if we consider the interval $[0,8]$. Then we need $8 n$ boxes of size $1 / n$. The common property of ome-dimensional intervals is that the number of boxes of size $\varepsilon$ required to cover an interval is no more than $C(1 / \varepsilon)$, where $C$ is a constant depending on the length of the interval. This proportionality is often expressed by saying that the number of boxes of size $\varepsilon$ scales $1 / \varepsilon$, meaning that the number of boxes is between $C_{1} / \varepsilon$ and $C_{2} / \varepsilon$, where $C_{1}$ and $C_{2}$ are fixed constants not depending on $\varepsilon$.

The square $\{(x, y): 0 \leq x, y \leq 1\}$ of side-length one in the plane can be covered by $n^{2}$ boxes of side-length $1 / n$. Any two-dimensional rectangle in $\mathbf{R}^{2}$ can be covered by $C(1 / \varepsilon)^{2}$ boxes of size $\varepsilon$. Similarly, a $d$-dimensional region requires $C(1 / \varepsilon)^{d}$ boxes of size $\varepsilon$. If we consider a square of side length 2 in the plane, and cover by boxes of side-length $\varepsilon=1 / n$, then $4(1 / \varepsilon)^{2}$ boxes are required, so $C=4$. The constant $C$ can be chosen as large as needed, as long as the scaling $C(1 / \varepsilon)^{2}$ holds as $\varepsilon$ goes to 0 .

We denote $N(\varepsilon)$ the number of boxes (Dindos, 2001) of side-length $\varepsilon$ needed to cover a given set. In general, if $S$ is a set in $\mathbf{R}^{m}$, we would like to say that $S$ is a $d$ dimensional set when it can be covered by

$$
N(\varepsilon)=C(1 / \varepsilon)^{d}
$$

boxes of side-length $\varepsilon$, for all small $\varepsilon$. Stated in this way, it is not required that the exponent $d$ be an integer. Let $S$ be a bounded set in $\mathbf{R}^{m}$. To measure the dimension of $S$, we lay a grid of $\boldsymbol{m}$-dimensional boxes of side-length $\varepsilon$ over $S$. Set $N(\varepsilon)$ equal to the number of boxes of the grid that intersect $S$. Solving the scaling law for the dimension $d$ gives us

$$
d=\frac{\ln N(\varepsilon)-\ln C}{\ln \left(\frac{1}{\varepsilon}\right)} .
$$


If $C$ is a constant for all small $\varepsilon$, the contribution of the second term in the numerator of this formula will be negligible for $\operatorname{small} \varepsilon$.

\section{Definition}

A bounded set $S$ in $\mathbf{R}^{n}$ has box counting dimension box $\operatorname{dim}(S)=\lim _{\varepsilon \rightarrow 0} \frac{\ln N(\varepsilon)}{\ln \left(\frac{1}{\varepsilon}\right)}$, where the lim it exists.

Computing the box-counting dimension of the Cantor middle $1 / 3$ set $\Gamma$

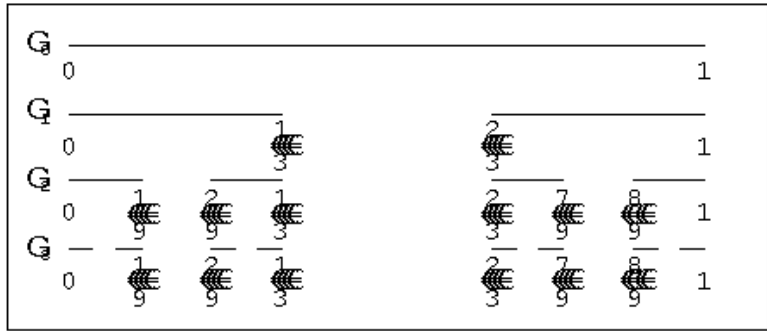

Fig. 1: Construction of the Cantor middle1/3 set

The set $\Gamma$ is contained in $\Gamma_{n}$ for each $n$. Just as $\Gamma_{1}$ consists of 2 intervals of length $1 / 3$, and $\Gamma_{2}$ consists of $2^{2}$ intervals of length $1 / 3^{2}$, and $\Gamma_{3}$ consists of $2^{3}$ intervals of length $1 / 3^{3}$, in general $\Gamma_{n}$ consists of $2^{n}$ intervals, each of length $\left(1 / 3^{n}\right)$. Further, we know that $\Gamma$ contains the endpoints of all $2^{n}$ intervals, and that each pair of endpoints lie $3^{-n}$ apart. Therefore, the smallest number of $3^{-n}$ boxes covering $\Gamma$ is $N_{0}\left(3^{-n}\right)=2^{n}$. We compute the box-counting dimension of the Cantor middle $1 / 3$ set $\Gamma$ as $\operatorname{Boxdim}(\Gamma)=\lim _{n \rightarrow \infty} \frac{\ln 2^{n}}{\ln 3^{n}}=\lim _{n \rightarrow \infty} \frac{n \ln 2}{n \ln 3}=\frac{\ln 2}{\ln 3}=0.63$.

Computing the box-counting dimension of the Cantor middle1/5 set $\Gamma$

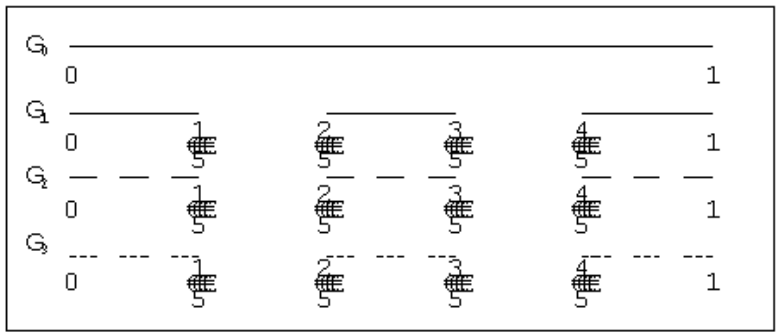

Fig. 2: Con struction of the Cantor middle1/5 set.
The set $\Gamma$ is contained in $\Gamma_{n}$ for each $n$. Just as $\Gamma_{1}$ consists of 3 intervals of length $1 / 5$, and $\Gamma_{2}$ consists of $3^{2}$ intervals of length $1 / 5^{2}$, and $\Gamma_{3}$ consists of $3^{3}$ intervals of length $1 / 5^{3}$, in general $\Gamma_{n}$ consists of $3^{n}$ intervals, each of length $\left(1 / 5^{n}\right)$. Further, we know that $\Gamma$ contains the endpoints of all $3^{n}$ intervals, and that each pair of endpoints lie $5^{-n}$ apart. Therefore, the smallest number of $5^{-n}$ boxes covering $\Gamma$ is $N_{0}\left(5^{-n}\right)=3^{n}$. We compute the box-counting dimension of the Cantor middlel $/ 5$ set $\Gamma$ as

$\operatorname{Boxdim}(\Gamma)=\lim _{n \rightarrow \infty} \frac{\ln 3^{n}}{\ln 5^{n}}=\lim _{n \rightarrow \infty} \frac{n \ln 3}{n \ln 5}=\frac{\ln 3}{\ln 5}=0.68$.

By the above, we can find the box-counting dimension of the Cantor middle $1 / 7,1 / 9,1 / 11, \Lambda$, in general, the Cantor middle $\frac{1}{2 m-1}$ set, where $2 \leq m<\infty$.

Computing the box-counting dimension of the Cantor middle $1 / 7$ set $\Gamma$

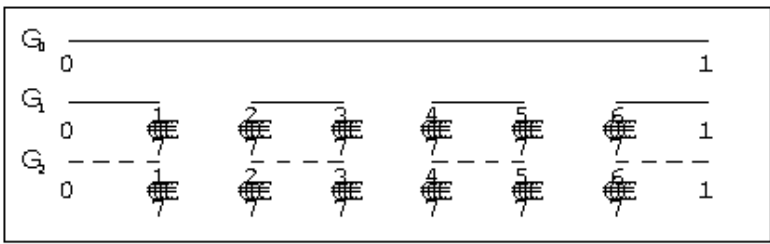

Fig. 3: Construction of the Cantor middle1/7 set.

The set $\Gamma$ is contained in $\Gamma_{n}$ for each $n$. Just as $\Gamma_{1}$ consists of 4 intervals of length $1 / 7$, and $\Gamma_{2}$ consists of $4^{2}$ intervals of length $1 / 7^{2}$, and $\Gamma_{3}$ consists of $4^{3}$ intervals of length $1 / 7^{3}$, in general $\Gamma_{n}$ consists of $4^{n}$ intervals, each of length $\left(1 / 7^{n}\right)$. Further, we know that $\Gamma$ contains the endpoints of all $4^{n}$ intervals, and that each pair of endpoints lie $7^{-n}$ apart. Therefore, the smallest number of $7^{-n}$ boxes covering $\Gamma$ is $N_{0}\left(7^{-n}\right)=4^{n}$. We compute the box-counting dimension of the Cantor middle $1 / 7$ set $\Gamma$ as

$\operatorname{Boxdim}(\Gamma)=\lim _{n \rightarrow \infty} \frac{\ln 4^{n}}{\ln 7^{n}}=\lim _{n \rightarrow \infty} \frac{n \ln 4}{n \ln 7}=\frac{\ln 4}{\ln 7}=0.71$. 


\section{Computing the box-counting dimension of the Cantor} middle1/9 set $\Gamma$

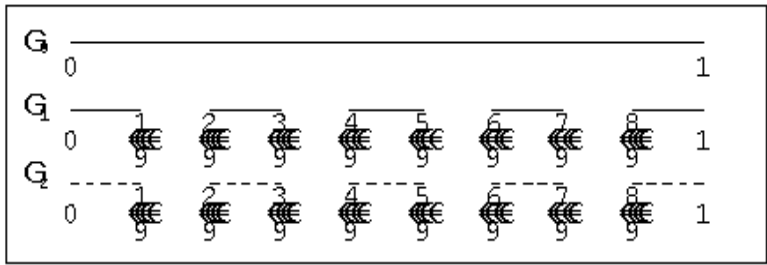

Fig. 4: Construction of the Cantor middle1/9 set.

The set $\Gamma$ is contained in $\Gamma_{n}$ for each $\boldsymbol{n}$. Just as $\Gamma_{1}$ consists of 5 intervals of length $1 / 9$, and $\Gamma_{2}$ consists of $5^{2}$ intervals of length $1 / 9^{2}$, and $\Gamma_{3}$ consists of $5^{3}$ intervals of length $1 / 9^{3}$, in general $\Gamma_{n}$ consists of $5^{n}$ intervals, each of length $\left(1 / 5^{n}\right)$. Further, we know that $\Gamma$ contains the endpoints of all $5^{n}$ intervals, and that each pair of endpoints lie $9^{-n}$ apart. Therefore, the smallest number of $9^{-n}$-boxes covering $\Gamma$ is $N_{0}\left(9^{-n}\right)=5^{n}$. We compute the box-counting dimension of the Cantor middle $l / 9$ set $\Gamma$ as

$\operatorname{Boxdim}(\Gamma)=\lim _{n \rightarrow \infty} \frac{\ln 5^{n}}{\ln 9^{n}}=\lim _{n \rightarrow \infty} \frac{n \ln 5}{n \ln 9}=\frac{\ln 5}{\ln 9}=0.73$.

Computing the box-counting dimension of the Cantor middle1/11 set $\Gamma$

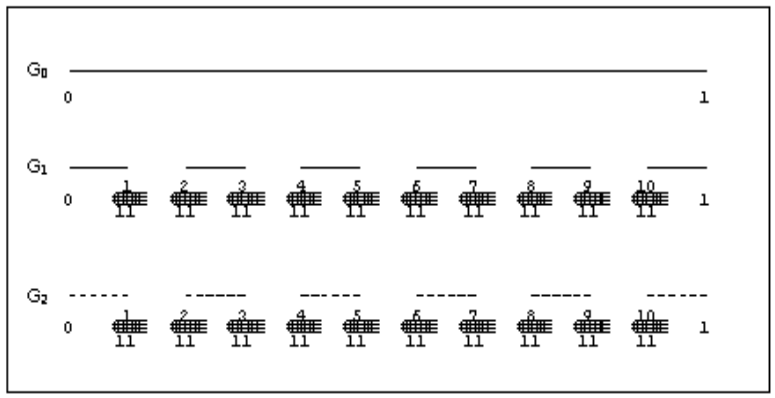

Fig. 5: Construction of the Cantor middle1/11 set.

The set $\Gamma$ is contained in $\Gamma_{n}$ for each $\boldsymbol{n}$. Just as $\Gamma_{1}$ consists of 6 intervals of length $1 / 11$, and $\Gamma_{2}$ consists of $6^{2}$ intervals of length $1 / 11^{2}$, and $\Gamma_{3}$ consists of $6^{3}$ intervals of length $1 / 11^{3}$, in general $\Gamma_{n}$ consists of $6^{n}$ intervals, each of length $\left(1 / 6^{n}\right)$. Further, we know that $\Gamma$ contains the endpoints of all $6^{n}$ intervals, and that each pair of endpoints lie $11^{-n}$ apart. Therefore, the smallest number of $11^{-n}$ boxes covering $\Gamma$ is $N_{0}\left(11^{-n}\right)=6^{n}$. We compute the box-counting dimension of the Cantor middle $1 / 11$ set $\Gamma$ as

$\operatorname{Boxdim}(\Gamma)=\lim _{n \rightarrow \infty} \frac{\ln 6^{n}}{\ln 11^{n}}=\lim _{n \rightarrow \infty} \frac{n \ln 6}{n \ln 11}=\frac{\ln 6}{\ln 11}=0.75$

In general, we will find the box-counting dimension of Cantor middle $1 /(2 m-1)$ set $\Gamma$, where $2 \leq m<\infty$. A similar Cantor set construction of Fig. 5 : can be altered to create the Cantor middle $1 /(2 m-1)$ set $\Gamma$. The set $\Gamma$ is contained in $\Gamma_{n}$ for each $n$. Just as $\Gamma_{1}$ consists of $\boldsymbol{m}$ intervals of length $1 /(2 m-1)$, and $\Gamma_{2}$ consists of $m^{2}$ intervals of length $1 /(2 m-1)^{2}$, and $\Gamma_{3}$ consists of $m^{3}$ intervals of length $1 /(2 m-1)^{3}$, in general, $\Gamma_{n}$ consists of $m^{n}$ intervals, each of length $1 /(2 m-1)^{n}$. Further, we know that $\Gamma$ contains the endpoints of all $m^{n}$ intervals, and that each pair of endpoints lie $(2 m-1)^{-n}$ apart. Therefore, the smallest number of $(2 m-1)^{-n}$ boxes covering $\Gamma$ is $N_{0}\left((2 m-1)^{-n}\right)=m^{n}$. We compute the box-counting dimension of the Cantor middle $1 /(2 m-1)$ set $\Gamma$ as

$$
\begin{aligned}
\operatorname{Boxdim}(\Gamma) & =\lim _{n \rightarrow \infty} \frac{\ln m^{n}}{\ln (2 m-1)^{n}}=\lim _{n \rightarrow \infty} \frac{n \ln m}{n \ln (2 m-1)} \\
= & \frac{\ln m}{\ln (2 m-1)}, \text { where } 2 \leq m<\infty
\end{aligned}
$$

\section{Conclusion}

We conclude the result as the generalized form of the Cantor set is the Cantor middle $\frac{1}{2 m-1}$ set, where $2 \leq m<\infty$, 
and the box-counting dimension of the Cantor set is $\frac{\ln m}{\ln (2 m-1)}$, where $2 \leq m<\infty$. If we increase the value of $m$, then the value of box-counting dimension of the Cantor middle $\frac{1}{2 m-1}$ set will be increased and tends to 1 .

\section{References}

Addison P. S. (1997). Fractals and Chaos, Institute of Physics, Bristol.

Dindos M. (2001). Generalized Cantor sets and sets of sums of convergent Alternating series, J. Appl. Ana., 7(1): 131-150.

Kathleen T. Alligood, Tim D. Sauer, James A. Yorke (1997). Chaos. An Introduction to Dynamical Systems, Springer Verlag.

Richard A. Holmgren (1996). A First Course in Discrete Dynamical Systems, Springer Verlag, New York,

Received: June 09, 2010;

Accepted: October 19, 2010 